\title{
X-Ray Images with Silicon Drift Detectors
}

\author{
R. Anderhalt, T. Nylese \\ EDAX Inc., 91 McKee Drive, Mahwah, NJ, 07430
}

The high X-ray throughput of the silicon drift detector (SDD) permits for the possible use of these detectors for imaging purposes [1]. There may well be a real need for X-ray images when analysis and mapping is done with a TEM that permits the deflection of the beam but does not allow the rapid scanning or rastering of the electron beam. It is possible to collect a map on such a system but the operator might not know the extent or boundaries of the map area unless they can collect a quick, preliminary X-ray image. This task is further complicated by relatively low X-ray count rates, commonly less than 10,000 counts per second. Another area where X-ray imaging capabilities would be useful is when an annular SDD is used. In this case, the SDD occupies the space normally occupied by, and precludes the effectiveness of, the traditional backscattered electron (BSE) detector. Other forms of compositional contrast would be quite useful and may be derived from the $\mathrm{X}$-ray data cube. In the latter situation, the X-ray count rates can be extremely high and the traditional secondary electron detectors would still be of use.

Aside from the elemental X-ray maps, the total X-ray counts image is probably the simplest and easiest of the X-ray images. The 'Counts' image can be compared to the BSE image (fig.1) and, at first glance, the contrast of the two images would appear to be quite similar. There are, however, some anomalies. There is a phase that is present in several locations but which is quite obvious in the lower right part of the BSE (fig. 1c) and of the 'Counts' (fig. 1d) image. It is darker than the surrounding area in BSE but brighter in the 'Counts' image. It might be possible to do some calculations with the data in the spectral map dataset to more closely approximate the contrast in the BSE image. An image has been calculated (fig. 2b) from a series of energy regions with a range of 2 $\mathrm{keV}$ (e.g. 0-2, 2-4, etc., up to 8-10 keV). In this calculation, a higher weighting factor has been given to the highest energy range image which is summed with each other energy range image -the weighting factor is reduced for each lower energy range. The weighting was originally determined as a means to compensate for the common reduction of counts at higher energies in the spectrum. The contrast of figures $2 \mathrm{a}$ (BSE) and $2 \mathrm{~b}$ ('Counts') are almost identical although it is clear that the X-ray image has more shadowing and shows less of the subtle effects of topography. Another possible approach is to use a color merge, either a red-green-blue (RGB) merge or a cyan-magenta-yellowblack (CMYK; figs. $2 \mathrm{c}$ and $2 \mathrm{~d}$ ) merge which are available in many readily available image editing programs. The difficulty with these color approaches is that they are limited to just 3 or 4 channels of information. The gray scale calculation does not have this limitation.

\section{References}

1. D. Newbury, Microsc. Microanal. 15 (Suppl 2) (2009) 666. 


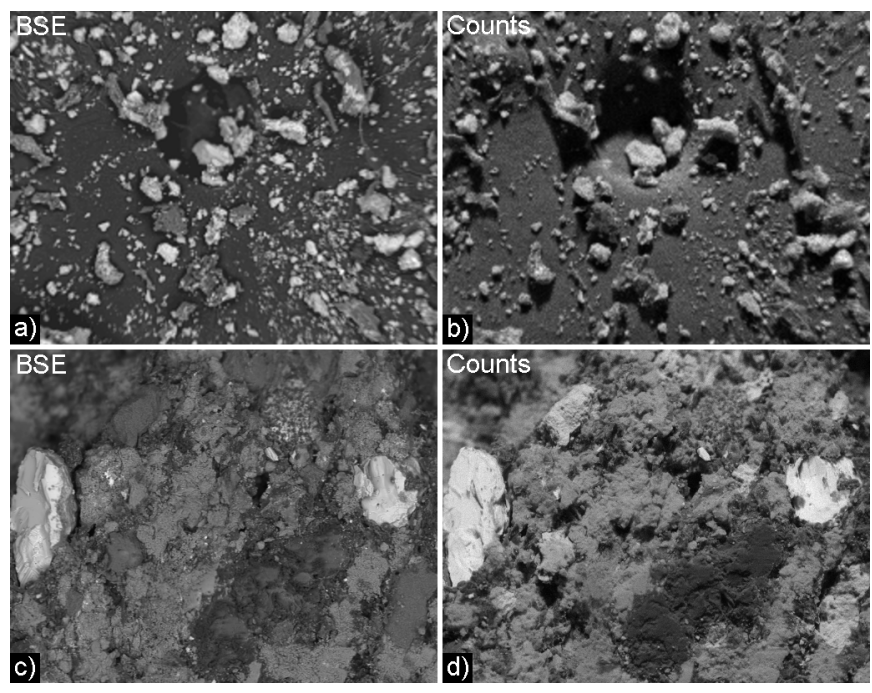

Fig. 1. Images of a multivitamin that show similarities and some dissimilarities between the BSE image and an image created from the total X-ray counts (labeled as "Counts"). In 1a and $1 \mathrm{~b}$ the multivitamin has been powdered and placed on carbon tape (image width $=0.79 \mathrm{~mm}$ ). In $1 \mathrm{c}$ and $1 \mathrm{~d}$ the pill has been fractured (image width $=1.4 \mathrm{~mm}$ ). The counts image has a similar contrast to the BSE image but there are some contrast anomalies. All images were collected with a beam voltage of $20 \mathrm{kV}$ in low vacuum mode (40 Pa).

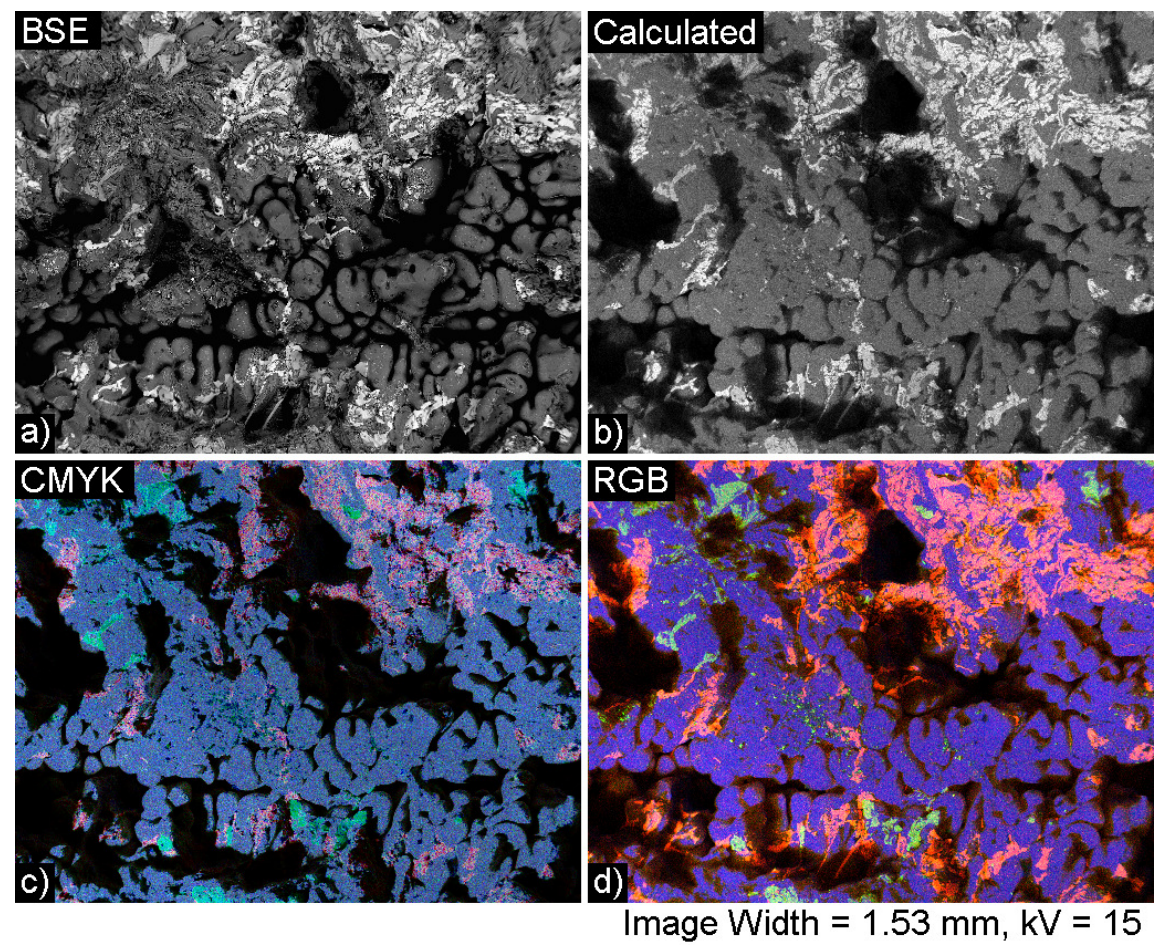

Fig. 2. A fracture surface of an aluminum alloy is shown in 4 different views from the same dataset. In $2 \mathrm{a}$ the BSE image can be seen. In $2 \mathrm{~b}$ through $2 \mathrm{~d}$ the X-ray data has been segmented into 5 energy ranges (0-2, 2-4, 4-6, 6-8 and 8-10 keV). In $2 \mathrm{~b}$ the image has been calculated such that the resultant image is more heavily weighted to the higher energies and progressively less weighted for the lower energies. The contrast is similar to the BSE image but has more shadowing and shows less subtle topographic variation. Figure $2 \mathrm{c}$ is labeled "CMYK" and the cyan (8-10 keV), magenta (6-8 keV), yellow (4-6 keV) and black $(0-2 \mathrm{keV})$ components have been merged to provide a contrast where blue represents the lower ' $Z$ ' phases, cyan to green for the middle ' $Z$ ' phases while the red to magenta areas represent the higher ' $Z$ ' phases. Figure $2 d$, "RGB", has its red channel (8-10 keV), green channel (6-8 keV) and blue channels (0-2 keV) merged to provide a similar contrast to the image in figure $2 \mathrm{c}$. 